\title{
Editorial
}

Vít Pokorný, Martin Nitsche*

\section{Philosophy and Sonic Research: Thinking with Sounds, Rhythms, and Music. An Editorial Introduction}

https://doi.org/10.1515/opphil-2020-0195

received November 29, 2021

Together with Bernd Herzogenrath, we can define the sonic thinking that we are trying to partially map with this issue as follows: "a thinking with and by means of sound, not a thinking about sound, which eventually does not deal with the question what music is, but rather what music can become. And from this vantage point, research and art, theory and practice, are coextensive." ${ }^{1}$ Thanks to all the participating authors, we managed to collect texts that do not only try to define sound, sonority, or music, but texts which are inspired by music and sound, which seek for philosophy's allies outside strict scholarly boundaries of philosophy - in art, in everyday experience, in technologies, and in nonhuman aspects of reality.

Therefore, we do not understand sound, sonority, and music only as objects of research. Encounters with this area of reality may also take a form other than theoretical. The Greek theory, of which we are still bearers and users, is connected with looking, with distance, with observation, grasping, measuring, determining. It is associated with the ocularcentrism of Western culture, with its emphasis on visuality, which implies a supervising, technocratic, and power-oriented attitude to the world. Thinking along with sound, through sonority, through or perhaps from within music and sound is associated with a different approach. With an attitude that favors listening, which is receptive and not controlling, for which meeting its subject has the nature of interaction and permeation, which does not understand sound as an object, but as an aspect of the environment in which we are immersed, which passes through us and thus shapes the very conditions of our presence in the world. The studies included in this issue therefore approach sonority in terms of various forms of sound and music practice - hearing and listening practice, everyday experience, music and sound practice, and only then, guided by sound experience and its structure, formulate theoretical statements about the nature of sound and methodological claims concerning the sonic thinking.

Martin Nitsche in his text attempts to situate sonic thinking within a phenomenological methodology. Here, based on a careful critical reading of Husserl's texts, he presents his topological-phenomenological interpretation of sonic environments. Nitsche shows that sounds are not some secondary qualities, that they are not appended to the things that produce them, nor are they just subjective experiences. According to him, the analysis of sonority opens up an access to a different understanding of the perceived environment than the one traditionally based on visuality. A sonic environment cannot be interpreted only objectivistically as composed of sound objects and events, nor only subjectivistically as a system of experiences. Instead, it should be conceived as a dynamic network of multisensory relationships developing in space, which is both a system of places and a system of localizations, i.e., movements, neighborhoods, and

1 Herzogenrath, Sonic Thinking, 9-10.

* Corresponding author: Martin Nitsche, Department of Contemporary Continental Philosophy, Institute of Philosophy, Praha 111000, Czech Republic, e-mail: nitsche.martin@gmail.com

Vít Pokorný: Department of Contemporary Continental Philosophy, Institute of Philosophy, Praha 111000, Czech Republic 
transformations. In this way, a sonic environment actually forms a medium within which sound events can appear.

Jiři Zelenka, Nitsche's doctoral student, analyzes the subjective pole of the auditory perceptual field, focusing on the difference between hearing and listening activities and asking whether it can be understood on the basis of the contrast between activity and passivity of phenomenological synthesis. If we understand hearing and listening not as subjective acts of consciousness but as a reversible dynamic relationship between the listening passivity of our exposure to the world and the listening activity of our intentional concentration, we have access to sonority as a dynamic field that is established in the intertwining of perceptual activities.

In his text, Ivan Gutierrez then thematizes the layer of sound environments that is connected with the auditory cultural practices and sound technologies. He does not understand technology naively as a system of things and procedures, but as a system of social relations that include subjects and objects, institutions, and events. Here, Gutierrez critically links post phenomenological accents with the actor-network theory (ANT) and existential hermeneutic interpretations. In today's societies, human sonic environments are fundamentally shaped technologically. From the point of view of media theory, it is true that specific communication medium creates specific forms of socio-cultural relations. The key to the creation of modern sonic environments was the invention of sound recording, which enabled the separation of sound and, together with other cultural practices, led to the creation of privatized media fields that are constantly connected to communication networks but in fact separate from others and from physical environments.

Vít Pokorný examines the lived environment in general from the perspective of rhythmanalysis. $\mathrm{He}$ connects rhythmanalysis with phenomenological analysis of rhythm and with deleuzo-guattarian processual thinking. On specific analyzes of the rhythmicity of the organism, the garden, and the city, he shows various modalities of the rhythmic formation of the living world, which go beyond the mere sonicity toward the more general principles of the structure of the environment. He does not understand rhythm as a linear sequence of beats, but as a general organizational principle. He understands rhythmically formed environments in accordance with Lefebvre's original rhythmic analysis as polyrhythmic bundles, i.e., as multilayered complexes of intertwined and interacting human and inhuman, natural and cultural rhythmical arrangements.

Following these phenomenological and post-phenomenological explorations, this topical issue contains another group of texts that are based on various types of experimental sound or music practices and that seek to explain various aspects of sound linked to these practices.

Lona Gaikis is amazed by the works of media artist Iona Moser called arboreal receptors and by the thinking of the Wood Wide Web team, which aims to "develop new technology enabling humans to increase their contact with nature by physically connecting people with trees and plants via the Mycorrhizal network or by sensors located directly on the tree/plants."2 In her philosophical interpretation, which is based primarily on the work of Susan Langer, and in general on Whiteheadian processual philosophy, she shows that in terms of today's experimental artistic approaches combined with an ecological perspective, the understanding of sonority cannot be based solely on human perception. Sound is for her a passageway for the synthesis of the self with the outside world. She therefore understands sound as something that transcends mere hearing and that takes the form of an imperceptible undercurrent. Trees do not respond to audio signals but can perceive undersonic vibrations. Such eco-phenomenological perspective reveals non-anthropomorphic patterns present in the constitution of our lived environments.

Vincenzo Zingaro reflects a different kind of music, resp. sound practice and, as for Gaikis, sonority means for him an area that transcends music and human perception and leads us to understand sound as an autonomous factor. The starting point of his thinking about the nature of sound is the emergence of a digital sampler and the emergence of sound design. Thanks to these technologies, sound has freed itself from its physical form, from hearing and haptics, and has become an infinitely transformable data event that can be manipulated using even visual technologies. From the perspective of digital sound design, the

2 http://woodwideweb.no/. 
sound is then converted to its very basic form, which is a sinusoidal wave common to every sound event. Zingaro then connects this basic view with Leibniz's contemplations about the nature of the monad and arrives at the notion of a sound monad, i.e., at an understanding of sound as a substance and the nucleus of both active and passive forces.

Edwin Østergaard, a composer and theorist, bases his research of sonority and sonic thinking on his own composing experience and, from this perspective, reflects on the relationship between listening and music. He convincingly shows that composing cannot be understood as purely intellectual creation, which is governed only by the will and intention of the composer. The composer's intentional creative activity is conditioned by the ability to listen to a musical work that is not yet finished: in its arrival it is no longer just a passive notation, but an active element to which the composer must tune. This knowledge is then the basis for a more general analogy, which concerns the human creative experience in general meaning that as humans we are not sole creators of meaning, because then only our voice would sound everywhere and the rest of the world would be silent. Real creation is a dialogue based on the relationship between the ability to hear and the ability to express.

The team of authors - Damián Keller, Luzilei Aliel, Marcos Célio Filho, and Leandro Costalonga - approach the thinking of the nature of music from the perspective of so-called Ubiquitous music. Ubiquitous music is a new area of sound research that combines ubiquitous computing, mobile and network music, eco-composition, and cooperative composition. The research presented in this article is a direct response to the changes that the Covid situation means for music creation and shared experience. The authors point to a conception of musicality that is limited neither by the idea of a written musical structure, nor by only traditional human musical activity. They do not see music as the ability to manipulate sound material in any way as something exclusively human, nor as something that only musicians do. Rather, they understand music as situated sonic information that can traverse a shared environment and can be based on a distributed and dispersed agency. Such a conception of musical practice can then be a model for a new understanding of shared culture and social interaction.

Another text based on the reception of own experimental practice is authored by Luca Soudant. She reflects on her artistic research and asks how sound design relates to the constitution of gender roles. She thinks of sound as a social and spatial event in relation to how sound can inhabit and territorialize the shared living space. In this context, she understands sonic manspreading as an activity where the sounds produced by males and male groups colonize the common space where women are symbolically and physically encouraged to be quiet. According to Soudant, the sonority of our corporeality and the sonority of the things we cope with are interconnected in the processuality of inter-corporeality and inter-sonority. In her experimental work, she shows that sounds are not only situated in perception, but that they pass through bodies and materials and connect the human with the inhuman. This mutual sound-based intertwining of all organic and inorganic bodies then opens the way to sonic thinking as a transformative event that allows us to approach newly the formation of our own identity.

Loiusa Collenberg also addresses the problem of the relationship between human and inhuman sonority, that is directly themed by Soudant and Gaikis, in her interpretation of D. Rothenberg's musical experiments, which take place on the borders of the human and animal realms. Rothenberg's musical improvisations cannot be understood as representations of animal sounds in music, nor as musical compositions inspired by animal sounds, but rather as the direct foundation of a musical/sound conversation between humans and non-human organisms. This approach calls into question not only the outdated boundaries between nature and culture, but specifically also the difference between an artistic creation and a natural sound expression. In fact, Rothenberg practically decentralizes the anthropocentric model of music, art, and culture with his improvisations and opens up space for a reassessment of the human place in the world.

Most of the texts published in this topical issue, in various ways, consider sonority and musicality as a counterweight to the preference of sight. Hannah Eßler and Jim Kallenberg speak in this context of "visualist ocularcentrism," "supremacy of the eye," "hegemony of vision," or "visual regime." Thinking through sound or music is then understood by the proponents of sound thinking as a way how to avoid this preference for distance and human, resp. male supervisors and replace it with another type of relationship to reality that is more receptive, more immersive, and that allows for a new approach in terms of new 
materialism, post-phenomenology, post-feminism, or critical theory. However, Eßler and Kallenberg point out that the enthusiasm of sonic thinking has a certain blind spot, which is the experience of the deaf. From the perspective of deafness, it is quite possible to speak of audio-centrism, a world that is intended primarily for the hearing. However, if we take the experience of the deaf seriously, not just as a shortcoming or disease, we get to the limits of sonic thinking. Eßler and Kallenberg examine these boundaries through the interpretation of the mythical meeting of Odysseus with the Sirens and call it sirenic thinking. This complex concept, in which sirens act both as a machine, as a myth, and as something human and inhuman, serves them to critique sonic thinking, which believes that by emphasizing sonority we can free ourselves from the visual metaphysics of the present, but which in reality confirms it.

In the final paper of the topical issue, Salomé Voegelin finds a possible way out of this stalemate in her project of singing philosophy. Her text undoubtedly represents the most radical and the most challenging, in terms of traditional philosophy, approach to sonic thinking in this issue. In it, Voegelin systematically encourages us not to think about music or sound, but to let sonority enter directly into the nature of philosophizing. Singing philosophy is supposed to break free from the hegemony of vision, which is based on comprehensibility and meaning, on the ability to measure and abstract. Instead, singing thinking should open up to the chaotic formation of a world that emerges only on the fringes of countable, normalized, and manageable. It should open up to a spontaneity in which there is no training, no standard, no predictability. Singing philosophy thus does not want to replace vision with hearing, instead it "[P]roduces the sonic time of music that creates a simultaneous space without bars or a meter. It invites us into a dark motility to dance not on the surface but within 'unconventional dimensions,' which we inhabit in sounding and listening and through which we can deviate from historical traditions and decolonialise philosophy's view, not by proposing another, but by seeing everything at once: traveling by Churten into a transiliential reality, whose margins never end." 3

Acknowledgements: The editors of the topical issue would like to thank the managing editor of Open Philosophy, Katarzyna Tempczyk, for her insightful and helpful approach to shaping the issue, to the editor-in-chief, Graham Harman, for his encouraging comments, to all anonymous reviewers for the thorough and critical reading of all manuscripts we received, and, last but not least, to our English language editor, Kyle L. Barbour, for his careful and precise work with our difficult texts.

\section{References}

Herzogenrath, Bernd (ed). Sonic Thinking: A Media Philosophical Approach. London, New York: Bloomsbury Academic, 2017. Voegelin, Salomé. “Singing Philosophy: Deviating Voices and Rhythms without a Time Signature.” Open Philosophy 4 (2021), 284-91. doi: 10.1515/opphil-2020-0186.

3 Voegelin, “Singing Philosophy,” 289. 